\title{
Optical fiber sensors: review of technology and applications
}

\author{
Mahmoud M. A. Eid \\ Department of Electrical Engineering, College of Engineering, Taif University, Taif, Saudi Arabia
}

\begin{tabular}{l} 
Article Info \\
\hline Article history: \\
Received Aug 28, 2021 \\
Revised Nov 23, 2021 \\
Accepted Dec 15, 2021 \\
\hline
\end{tabular}

Keywords:

Automation

Digital transformation

Internet of things

Optical sensors

Smart cities

\begin{abstract}
There is a huge increase in the usage of optical fiber sensors in various fields, especially the field of communications, as these sensors have been employed in a promising industry, namely, the internet of things. This industry is witnessing a growing demand for more sensors as well as employing them in new applications, and there is an urgent need to invent new sensors to meet our requirements in providing more time, luxury, and effort with the highest quality and the best possible performance. But there is now a lot of information about optical sensors as well as many classifications and applications. There are also some developments in a scientific research yard. The main objective of this paper is to introduce short, effective, and concentrated points in optical fiber sensors such as a brief historical background, their structure, and their different operation principles, different classifications for these sensors according to different categories, and finally advantages of fiber optical sensors compared to traditional electronic sensors. I hope this content will be very useful to anyone interested in these types of sensors. This review is done with particular assurance on the recently published information.
\end{abstract}

This is an open access article under the CC BY-SA license.

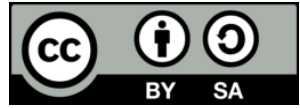

\section{Corresponding Author:}

Mahmoud M. A. Eid

Department of Electrical Engineering, College of Engineering, Taif University

Taif, Saudi Arabia

Email: m.elfateh@tu.edu.sa

\section{INTRODUCTION}

Recently the amount of data being exchanged across the world is increasing. The main objective of all of this is to provide means of security and speed of decision-making that contribute effectively and directly to the proper planning processes in various areas of life. One of the most important tools that provide us with fast, timely, and continuous data are sensors. By studying and analyzing the data collected from these sensors, we can track and monitor changes that occur in various aspects of our lives. For example, but not limited to, temperature, humidity, and gas sensors provide much information about the climate, which contributes to inferring the causes of changes that occur in the environment. Medical sensors provide information about the vital signs of individuals, which contributes to their medical diagnosis and reduce the spread of epidemics. Also, data may be collected through social media about persons to understand their behavior and interests and adapt this in the marketing of some companies' products. Motion detection sensors and thermal sensors provide data on activities and changes or movements that take place in a specific environment and employ this in maintaining the security system. There are also many sensors that are employed in fields of industry and civil construction. There is no doubt that digital transformation and the general tendency to create smart cities, automate systems and employ the internet of things (IoT) in many vital areas in various aspects of our lives such as health care, agriculture, environment, transportation, industry, and security, led to saving time, effort, and money. By employing these technologies, the most appropriate decisions in the least possible time could be taken by the decision-maker [1]-[7]. 
The perfect solution to all of this is sensing engineering and science. They are considered the main components of modern technology that is currently used in many engineering applications in most aspects of life such as control of monitoring, security, and safety processes in industrial, military, and aerospace applications, as well as in monitoring, diagnostics, and awareness in most medical applications. Therefore, both technicians and scientists need to understand how sensors work, the physical principles of how they work as well as their properties, and how they can be used in those different applications [8], [9].

Here it is worth mentioning (M2M), the machine to machine, human to machine, or a machine to man technology. M2M is combined with modern communication systems to create a system characterized by intelligence, continuous and instantaneous interaction. This interaction occurred between the sensors used and the rest of the system parts, this is what is referred to as (IoT) [3], [10].

It is possible to include the most important applied fields of these sensors so far under the following points:

a) Wearable devices like smart clothing, smartwatches, fitness tracker, wearable medical device, or healthcare monitoring systems.

b) Smart homes applications like smart locks, sensors for different parameters such as motion, gas, pressure, temperature, sound, or ambient light.

c) Smart vehicles which including unmanned aerial vehicle (UAV) such as drones, unmanned underwater vehicles (UUV), and applications for industrial automation and military and space applications [10]-[14].

\section{RESEARCH METHOD}

One of the most effective and promising and most requested sensors in recent times are optical fiber sensors (OFSs) have been shown up in most aspects of our life and are widely used in the digitalization of automation systems, IoT, and smart cities. So, there are important points that should be mentioned in the method section first, the definition, and a historical perspective of OFSs will be reviewed depending on recent references. After that, the design and the operation principle of OFS will be mentioned. The main components of OFS and the main role of each component will be explained very clearly. Then a simple and comprehensive classification for OFSs will be listed. Finally, some advantages of FOSs compared to traditional electronic sensors will be listed.

\subsection{Definition and a historical perspective of optical fiber sensors}

OFSs are considered a remotely powerful tool. They are used for collecting information from the surrounding medium by detecting and analyzing light characteristics at specified electromagnetic spectrum ranges. This spectrum includes different regions such as ultraviolet (UV), infrared (IR), and visible regions. Where one or more light propagation characteristics (such as wavelength, frequency, amplitude, phase, intensity, polarization) will be affected by the measurement of physical, chemical, or biological phenomena [3], [5], [15]-[18].

More than 60 years had been passed in developing OFSs. The main reason for the relative delay in the prosperity of the manufacture of these sensors and the spread of their applications is the urgent need for advanced technologies to manufacture them, as well as the manufacture of distinguished light sources, modulators, waveguides, and detectors. In early 1961 the term optical sensors first appeared in a publication of the optical society of America (OSA). At the beginning of the 1980s, researchers were interested in the technology of interference fiber optic sensing. After that in 1986, the OFS network (OFSN), distributed and quasi-distributed optical sensors had emerged, and at the end of the 1980s, the researchers focused on the fiber bragg gratings (FBG) technology and its important role in the progress of the fabrication of OFSs, which led to the appearance of new products such as optical radar, which depends on optical time-domain reflectometer (OTDR). Also, raman distributed temperature sensors (RDTSs) had appeared. At the end of the 90s, techniques of Brillouin sensing represented in Brillouin optical time-domain reflectometry (BOTDR) and Brillouin optical time-domain analysis (BOTDA) became commercially available. After the beginning of 2000, there were a boom in optoelectronic devices and signal processing technologies. All of these evolutions led to the discovery of new applications for different sensors, such as fabry-perot interferometric (FPI) OFSs, FBG sensors, and distributed OFSs. Now at the beginning of the 2000s, there is a promising new optical fiber called "photonic crystal fibers" that contribute to the development of the biophotonics scientific sectors, also to develop and innovate new devices in the industry sector, like wideband optical sources, and new sensors [19]-[24].

\subsection{Optical fiber sensor design and the operation principle:}

When managing any system or monitoring a phenomenon many perturbations or is called measurands will be noticed. There are many different types of perturbations i.e., physical, chemical, or biological perturbations. For clarification, the physical attribute (such as force, pressure, strain, temperature, speed, distance, acceleration, and torque) could result from an instrument, apparatus, equipment, or 
machines, and any other systems. So, we will need an electronic integrated system to detect these disturbances and provide some important data about some variables to be measured or follow-up to their change constantly and give other devices these data as feedback to take suitable actions. This will maintain the stability of the system. To do those different types of devices which are called sensors we will be used.

OFS is one of the most important and promising types of sensors. Since the light properties such as intensity, frequency, wavelength, and polarization are affected by most disturbances, then the OFS will detect them. This means that the operation principle of the OFS based on the parameter of the optical light source that will be modified by the outer perturbations. Now the working flow of the optical sensor will be described in the following steps as shown in Figure 1.

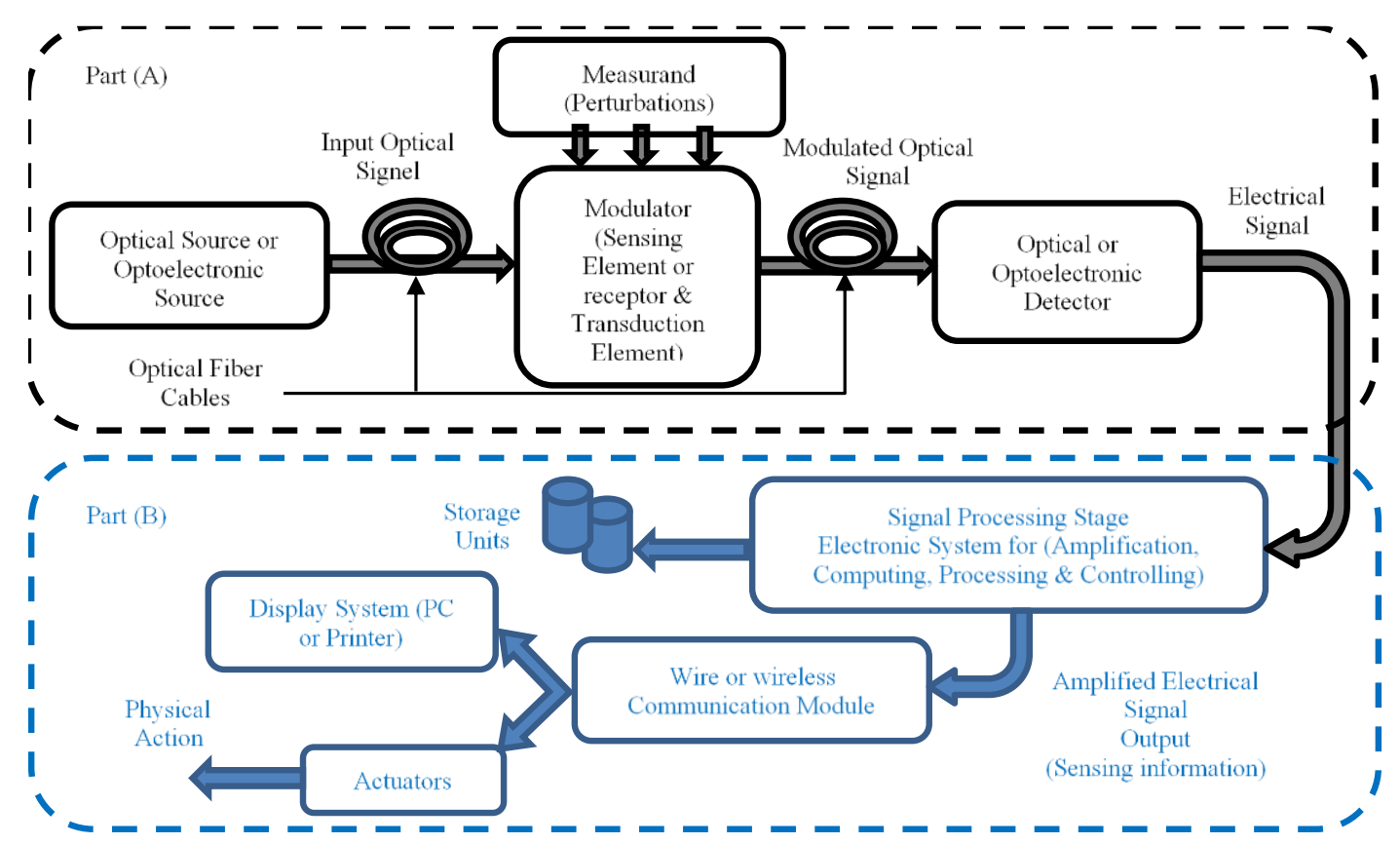

Figure 1. Schematic diagram of the sensor (part A) integrated with an edge of intelligence (part B)

First, the optical light source also called the optoelectronic source injects the light in one end of the optical fiber cable, this light traveling through this cable until reaches the modulator which consists of a sensing element and transduction element. In the modulator, there will be a change in the properties of the input optical signal because of the surrounded measurand, or maybe called perturbations. This means at least one of the following properties of the optical signal such as amplitude, phase, color (spectral signal) will be changed. The measured parameters of the modulated optical signal will be obtained as an output of the modulated stage. Now, the output is a modulated optical signal which will travel through another optical fiber cable until reach the optoelectronic detector which produces an output signal in a suitable format for the edge of the intelligence stage that including some devices like controller which is based on microprocessor/ microcontroller to compute/process the received data, after that, a copy of these data will be saved on a storage unit, and at the same time a copy will be sent to wire or wireless module to communicate with actuators which is responsible on the physical actions which will be taken or will send a signal to the display system to display and monitor the perturbations [3], [19]-[22], [25]-[34].

\subsection{Main optical fiber sensor components}

From the previous section, it is very clear that the main parts of the optical sensor construction are three parts: optical source, modulator, and the optical detector. As a result of the tremendous development, scientific leaps in modern branches of science (such as nanotechnology and artificial intelligence), and the continuous development in modern communication systems especially optical communication systems, all these developments have led to the creation of advanced and modern optical sensor systems and components. Below we discuss the main components of OFS [5], [31], [32].

\subsubsection{Optical source}

There are many different light sources were used in the industry of the OFSs. Some of them are listed: 
- $\quad$ Light emitted diode (LED): low cost and available in always more spectral range from UV to infrared using a new semiconductor.

- $\quad$ Superluminescent diode (SLD): more powerful than a classical LED. More generally, a lot of progress have allowed increasing the LED output power.

- The coherent light amplification by stimulated emission of radiation diode (LASER-D): available in always more spectral range from UV to infrared, its cost has decreased, and its power could be controlled.

- Vertical-cavity surface-emitting laser (VCSEL): is more powerful than classical LASER-D but less coherent, and is often multimode [5], [32].

\subsubsection{Optical fiber}

Recently there are many new designs, structures, geometries, and new dopants materials for the fabrication process of optical fiber cables. So, there are now many different types of optical fiber cables, such as single-mode and multi-mode structures with different core diameters dimensions from few micrometers to few millimeters. Some of them are listed.

- $\quad$ Step index and graded-index fiber could be made by using several different indices.

- $\quad$ Polarization maintaining fiber (PMF) has different designs for making birefringence in the fiber. The main three types of PMF are elliptical clade or is called oval-inner clad fibers, PANDA chape, and bow-tie.

- Photonic crystal fiber (PCF), is a promising type of microstructure fiber in the direction of radiation.

- Fibers are fabricated from different materials such as polymer or silicate (plastic), or silica which is doped with different dopant materials like (fluorine, erbium, germanium, and alumina).

- $\quad$ Bragg grating fiber which its refractive index is regularly modulated along its length [5], [32].

\subsubsection{The optical modulator}

To get acquainted with this very important part of optical sensors, we must also know its working principle and its different types. This part is responsible for transmitting various influences from the surrounding environment to be monitored. As we mentioned previously the external influences affect the optical properties and the way the light propagates within the optical fiber. That is why it is so important to choose the right modulator (which containing the sensing and transduction elements) to be sensitive to the required external influence or phenomena which want to be detected and monitor. This point will be covered in the section of classification of the OFS [5].

\subsubsection{The optical detectors}

Because of existing of many different types of OFSs, it is reasonable that there will be also different types of optical detectors, which will respond only to the optical energy, this energy is related to the optical parameters of the light wave such as amplitude, phase, polarization, and frequency. Each specified type of OFS has its need to detect the perturbations depending on the modulated optical parameters which will be measured. There are varieties of optical detectors such as a photodiode (PD), avalanche photodiode (APD), and a photomultiplier tube (PMT). All of these could be individually or integrated with other devices. For example, photodiode could be used directly for intensity measurement or could be used with other devices such as polarizer and wave plates for determining the polarized state [5], [23], [35].

\subsection{Classification of the optical fiber sensor}

There are many ways to classify the OFSs. The most comprehensive classification is done by classifying the OFSs into three main categories. These categories are based on the location of the sensors, the operating principle, and the last one according to its application, as listed blew in Table 1 [20], [25], [27], [28], [32], [36]-[38].

Table 1. Classifications of the FOSs according to three categories

\begin{tabular}{cl}
\hline Category & \multicolumn{1}{c}{ Types } \\
\hline Sensing Location & Point Sensors \\
& Distributed Sensors \\
& Quasi-distributed Sensors \\
Operating Principle & Intensity Sensors \\
& Phase Sensors \\
& Frequency/Wavelength Sensors \\
& Polarization Sensors \\
Application & Physical Sensors \\
& Chemical Sensors \\
& Bio-medical Sensors \\
\hline
\end{tabular}




\subsubsection{Based on the sensing location}

The general classification for this category has two types, intrinsic or extrinsic OFS. The difference between these types is shown in Figure 2. In an intrinsic OFS, the sensing element is a part of the optical fiber itself, and light is propagating inside the fiber and does not go out of it, where the external perturbation modulates the transmitted light by changing one or more from its parameters. But in the extrinsic OFS, the sensing element is a separated part from the optical fiber, so the fiber is only used in this case to transmit the light to this external sensing element and inside the sensing element, the light is modulated [20], [25], [27], [28], [32], [36], [39], [40].

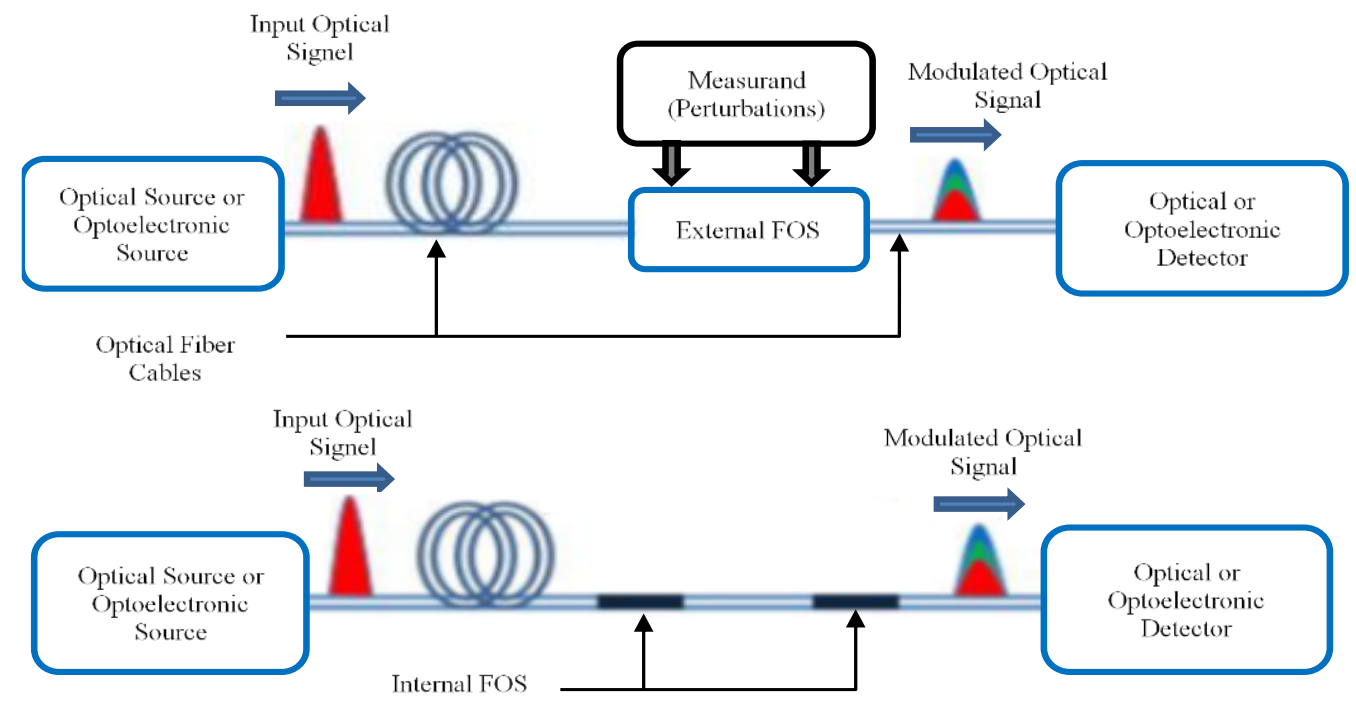

Figure 2. Difference between (a) extrinsic and (b) intrinsic OFSs

Also, there is another classification according to OFSs distribution along with the optical fiber. So OFSs can be classified into major three subsets:

a) Single point, only one sensor is existing as shown in Figure 3(a).

b) Multi-point or called (quasi-distributed), multi-sensor is existing as shown in Figure 3(b).

c) Distributed or called (continuous sensing element) OFSs, the OFSs are distributed continuously along the optical fiber cable as shown in Figure 3(c) [20], [25], [27], [28], [32], [36], [37].

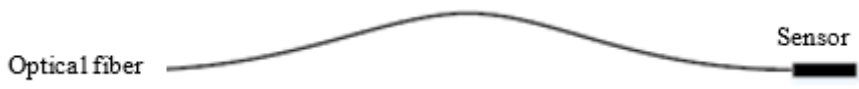

(a)

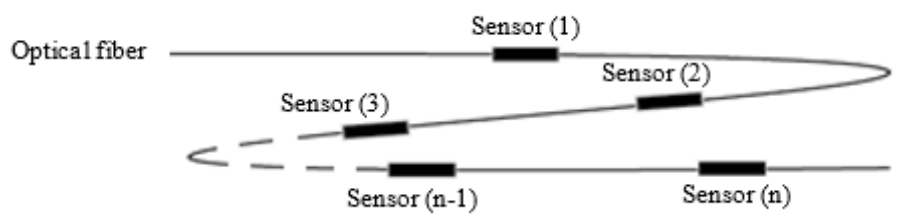

(b)

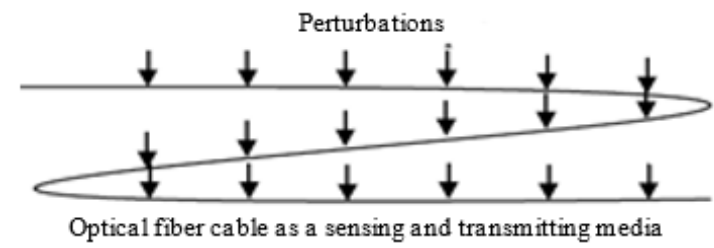

(c)

Figure 3. Distribution types of OFSs: (a) point-based type, (b) quasi-distributed type, and (c) distributed type 


\subsubsection{Based on the operating principle}

The light has a duality property, it consists of particles called photons, or it is electromagnetic waves, in which the electric and magnetic fields are perpendicular to each other, and to the direction in which the light travels. It has been mentioned previously that the operation principle of OFSs depends on the modulation occurs for the transmitted light, and this modulation is a result of the external perturbations, which causing a change in one of the optical parameters such as intensity, phase, frequency, or polarization of the transmitted light. So, the OFSs can have another classification depending on the parameter which is changed due to the influence of the external perturbations. The mathematical interpretation for these different modulated optical parameters is shown in Figure 4. From all information in this section, we can summarize the main four types of OFSs according to the operation principle in the following points [23], [27], [40].

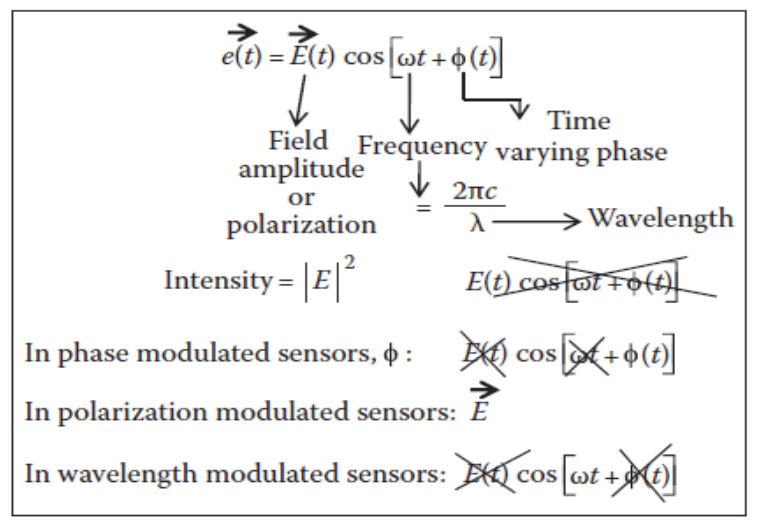

Figure 4. Mathematical interpretation for these different modulated optical parameters

a) Intensity (or Amplitude) based OFSs

The external influences in the environment could be detected by measuring the variation in the intensity of transmitted light. There are many techniques to measure the intensity, but the main three intensity-modulated sensors are transmissive, reflective, and macro/micro bend type. Many different optical components could be used such as lenses, mirrors, prisms to direct the light to the desired point of measurement. This is also done with the help of several other optical devices such as beam splitters, isolators, connectors, and couplers. Also, multiplexing techniques could be used if there are multipoint of sensors [23], [27], [28], [32].

b) Phase-based OFSs

This type of OFS is based on phase modulation and is more accurate and more sensitive than intensity modulation. The main device which is used to detect the change in the phase is called an interferometer. The most frequently used are Mach-Zehnder, Fabry-Perot, Michelson, polarimetric, Sagnac, and grating interferometers. Its operation principle depends on comparing the phase of coherent light after splitting it and making an injection into two single-mode fibers, one will be used as a reference and the other will be exposed to the external perturbations which will cause change or shift to the phase of the light signal. It is noteworthy that, the phase variation will lead to a change in frequency and wavelength of the optical signal [23], [27], [28], [32].

c) Frequency/wavelength based OFSs

This type of OFS depends on detecting the change in frequency or wavelength of the transmitted light. This change is caused by external perturbation. This type of OFS has different compositions which are used in many fields, such as fluorescent FOS which is used in the medical field, and FBG sensor which depends on granting of the refractive index periodically [28].

d) Polarization based OFSs

The light is an electromagnetic wave that consists of electric and magnetic fields perpendicular to each other, and to the direction in which the light travels. If the electric field variation is located in one plane, then the light is called polarized light. There are three main types of polarized light, linear, elliptical, and circular polarization. But if the vibrating of the electric field is in more than one plane, then the light is unpolarized. So, polarization is the way that describes continuously the electric field propagation as a function in time. The operation principle of polarization-based OFSs depends on the influence of external perturbation such as stress or strain on the refractive index of the fiber, which is called in this case induced refractive index. Any change in the refractive index will lead to a change in polarization directions and as 
result, there will be a significant phase difference. Therefore, the external perturbation can be sensed by detecting the change in the polarization [5], [23], [27], [28], [32], [41].

\subsubsection{Based on the operating applications}

FOSs could be used in difficult circumstances when the ordinary electronic and electrical sensors having trouble working in these conditions. The following Table 2 summarized the description for different types of OFSs based on their usage in different fields according to the operating applications [20], [25], [27], [28], [32], [36], [37], [42]-[47].

Table 2. Classifications of the FOSs according to the operating applications

\begin{tabular}{|c|c|c|c|}
\hline & \multicolumn{3}{|c|}{$\begin{array}{l}\text { OFSs Based on the Operating Applications } \\
\text { Chemical sensors }\end{array}$} \\
\hline Description & $\begin{array}{l}\text { Physical sensors are widely } \\
\text { used for monitoring and } \\
\text { controlling many industrial } \\
\text { processes. }\end{array}$ & $\begin{array}{l}\text { Optical fiber chemical sensors (OFCSs) } \\
\text { are a small version of analytical devices, } \\
\text { which provide the required information } \\
\text { about the assistance of certain ions or } \\
\text { compounds in complex samples. }\end{array}$ & $\begin{array}{l}\text { Optical fiber Bio-medical sensors } \\
\text { (OFBSs) are Optoelectronic devices } \\
\text { converting the biological signals into } \\
\text { measurable electric signals, which are } \\
\text { used in medical diagnostic. }\end{array}$ \\
\hline $\begin{array}{l}\text { Some } \\
\text { Usage }\end{array}$ & $\begin{array}{l}\text { Measuring physical properties } \\
\text { such as force, pressure, strain, } \\
\text { temperature, speed, distance, } \\
\text { position, acceleration, torque, } \\
\text { and radiation. }\end{array}$ & $\begin{array}{l}\text { Used in spectroscopy, gases and smokes } \\
\text { analysis, air pollution, groundwater } \\
\text { contamination, soil contamination, and } \\
\text { the concentration of a given compound } \\
\text { such as pH. }\end{array}$ & $\begin{array}{l}\text { Measurement of blood flow, blood } \\
\text { pressure, temperature } \mathrm{Oxygen} \text { level in the } \\
\text { blood, measuring of } \mathrm{O}_{2}, \mathrm{CO}_{2} \text {, and } \mathrm{PH} \text { at } \\
\text { the same time, glucose content, and } \\
\text { measure the pressure of intracranial. }\end{array}$ \\
\hline
\end{tabular}

\subsection{Advantages of FOSs compared to traditional electronic sensors}

FOSs have several advantages over traditional sensors, so they are being developed and increased its scope of applications. Among the most prominent and recent applications are space science, biotechnology, and law enforcement security applications such as digital imaging. All this has increased the demand for these sensors. Among the most prominent features of optical sensors, for example, are the following points [3], [5], [17], [20], [21], [26]-[30], [32], [43], [48]-[50].

\subsubsection{Advantages from point of design}

- It is lighter in weight, less bulky, less energy-consuming, and has a longer life span, which makes its manufacturing cost low compared to other types of sensors.

- The quality of performance as it has high sensitivity, and can be easily combined with several devices, whether electronic, mechanical, and or medical.

- It is characterized by its high immunity to electromagnetic interference (EMI), so it is widely used in high voltage applications.

- High immunity to corrosion so it is employed in corrosive environments.

- $\quad$ Its ability to work in harsh environments and at elevated temperatures.

- Flexibility in design, which allows the measurement of a myriad of physical, chemical, biological, and other variables.

\subsubsection{Advantages from point of communication system}

- It has a wide bandwidth.

- The availability of the possibility of multiple transmission, which gives great flexibility in the possibility of providing instantaneous and continuous monitoring of the environmental disturbances to be monitored.

- The signals from these sensors are transmitted over long distances because the optical fibers are characterized by very low attenuation coefficients compared with other transmission media. This feature allows the possibility of remote sensing, especially in hard-to-reach areas.

- The ability to store and analyze data and then use it to make optimal decisions.

\section{CONCLUSION}

In this review study, we illustrate the importance of OFSs, their definition, main components, operation principles, and classified them according to different categories Also, we mentioned the advantages of OFSs compared to traditional electronic sensors. Also, we should mention here that there are many prospects of the development of OFSs in many application areas in further studies if we search about the problems and obstacles that we still suffer from around the world in various areas related to our lives like climate. All this 
comes with many challenges to maintain security and privacy. This makes sense because of increasing our fears of complete dependence on modern technologies in various aspects of our life and taking into account negative effects on some factions of society as a result of the trend to employ modern technologies. The negative effects such as increasing unemployment rates, and the speed of rampant misinformation. Countries now rely on data in planning for the future and based on accurate studies to a large extent. Accurate data leads to the optimal use of country resources, as well as preserving the environment to provide a better climate for its citizens, which contributes to reducing spending on sectors such as health, and we have seen this during the Corona pandemic since late 2019. From all of the above, it becomes clear to us the importance of sensors in providing the information that we need on time.

\section{REFERENCES}

[1] L. Belli et al., "IoT-Enabled Smart Sustainable Cities: Challenges and Approaches," Smart Cities, vol. 3, no. 3, pp. 1039-1070, 2020, doi: 10.3390/smartcities3030052.

[2] P. Chamoso, A. González-Briones, S. Rodríguez, and J. M. Corchado, "Tendencies of Technologies and Platforms in Smart Cities: A State-of-the-Art Review," Wirel. Commun. Mob. Comput., vol. 2018, p. 3086854, 2018, doi: 10.1155/2018/3086854.

[3] W. Zeng and H. Gao, "Optic Fiber Sensing IOT Technology and Application Research," Sensors \& Transducers, vol. 180, no. 10, pp. 16-21, 2014, October 2014

[4] X. Shi and D. Kong, "Application of IOT Technology and Remote Control Technology in Industrial Automation Control," 2015 International Conference on Computational Science and Engineering, pp. 342-345, 2015, doi: 10.2991/iccse-15.2015.63.

[5] S. Lecler and P. Meyrueis, "Intrinsic Optical Fiber Sensor," In Fiber Optic Sensors, pp. 53-76, 2012, doi: 10.5772/27079.

[6] R. Harmon, E. Castro-Leon, and S. Bhide, "Smart cities and the Internet of Things," Portland International Conference on Management of Engineering and Technology (PICMET), pp. 485-494, 2015, doi: 10.1109/PICMET.2015.7273174.

[7] H. Khlaifi, A. Zrelli, and T. Ezzedine, "Optical fiber sensors in border detection application: Temperature, strain and pressure distinguished detection using fiber Bragg grating and fluorescence intensity ratio," Optik (Stuttg)., vol. 229, p. 166257, 2021, doi: 10.1016/j.ijleo.2021.166257.

[8] G. R. Sinha, “Advances in Modern Sensors," Physics, design, simulation and applications. IOP Publishing, pp. 20-21, 2020, doi: 10.1088/978-0-7503-2707-7.

[9] V. Alcácer and V. Cruz-Machado, "Scanning the Industry 4.0: A Literature Review on Technologies for Manufacturing Systems," Eng. Sci. Technol. an Int. J., vol. 22, no. 3, pp. 899-919, 2019, doi: 10.1016/j.jestch.2019.01.006.

[10] M. Stoyanova, Y. Nikoloudakis, S. Panagiotakis, E. Pallis, and E. Markakis, "A Survey on the Internet of Things (IoT) Forensics: Challenges, Approaches and Open Issues,” IEEE Commun. Surv. Tutorials, p. 1, Jan. 2020, doi: 10.1109/COMST.2019.2962586.

[11] A.-V. Emilien, C. Thomas, and H. Thomas, "UAV \& satellite synergies for optical remote sensing applications: A literature review," Sci. Remote Sens., vol. 3, 2021, doi: 10.1016/j.srs.2021.100019.

[12] O. Shapira et al., "Implementation of a Fiber-Optic Sensing Technology for Global Structural Integrity Monitoring of UAVs," The 54th Israel Annual Conference on Aerospace Sciences, 2014.

[13] F. S. Delgado, J. P. Carvalho, T. V. N. Coelho, and A. B. Dos Santos, "An Optical Fiber Sensor and Its Application in UAVs for Current Measurements," Sensors (Basel)., vol. 16, no. 11, p. 1800, Oct. 2016, doi: 10.3390/s16111800.

[14] K. Annamdas and V. G. Annamdas, "Review on Developments in Fiber Optical Sensors and Applications," Proc. SPIE, vol. 7677, p. 76770R, Apr. 2010, doi: 10.1117/12.849799.

[15] M. Asirdizer, Y. Hekimoglu, and O. Gumus, "Usage of Infrared-Based Technologies in Forensic Sciences," in Forensic Analysis - From Death to Justice, pp. 97-118, 2016, doi: 10.5772/62773.

[16] M. Zeeshan, R. R. Deshmukh, and S. B. Syed, "An Overview of Different Optical Remote Sensing Techniques," Int. J. Eng. Adv. Technol., vol. 6, pp. 181-183, Mar. 2017.

[17] M. F. S. Ferreira et al., "Roadmap on optical sensors," J. Opt., vol. 19, no. 8, Aug. 2017, doi: 10.1088/2040-8986/aa7419.

[18] X. Lu, P. J. Thomas, and J. O. Hellevang, "A Review of Methods for Fibre-Optic Distributed Chemical Sensing," Sensors, vol. 19, no. 13, 2019, doi: 10.3390/s19132876.

[19] B. Culshaw and A. Kersey, "Fiber-Optic Sensing: A Historical Perspective," Light. Technol. J., vol. 26, pp. 1064-1078, Jun. 2008, doi: 10.1109/JLT.0082.921915.

[20] J. Castrellon-Uribe, “Optical Fiber Sensors: An Overview,” pp. 1-26, 2012, doi: 10.5772/28529.

[21] H. Qiu, F. Min, and Y. Yang, "Fiber optic sensing technologies potentially applicable for hypersonic wind tunnel harsh environments," Adv. Aerodyn., vol. 2, no. 1, p. 10, 2020, doi: 10.1186/s42774-020-00033-y.

[22] P. Ferdinand, "The Evolution of Optical Fiber Sensors Technologies During the 35 Last Years and Their Applications in Structure Health Monitoring," EWSHM-7th European Workshop on Structural Health Monitoring, Jul. 2014.

[23] T. Yoshizawa, Handbook of Optical Metrology: Principles and Applications, Florida, USA: CRC Press, 2009.

[24] D. Pallarés-Aldeiturriaga, P. Roldán-Varona, L. Rodríguez-Cobo, and J. M. López-Higuera, "Optical Fiber Sensors by Direct Laser Processing: A Review," Sensors (Basel)., vol. 20, no. 23, Dec. 2020, doi: 10.3390/s20236971.

[25] V. Naresh and N. Lee, "A Review on Biosensors and Recent Development of Nanostructured Materials-Enabled Biosensors," Sensors, vol. 21, no. 4. 2021, doi: 10.3390/s21041109.

[26] A. Lobnik, M. Turel, and S. K. Urek, "Optical Chemical Sensors:Design and Applications," Advances in chemical sensors, pp. 3-28, 2012, doi: 10.5772/31534.

[27] P. Khandelwal, "Optical Fiber Sensors: Classification \& Applications," International Journal of Latest Technology, Managenment \& Applied Science, vol. II, no. Vii, pp. 22-25, 2013.

[28] H. Hisham, "Optical Fiber Sensing Technology: Basics, Classifications and Applications," Am. J. Remote Sens., vol. 6, Feb. 2018, doi: 10.11648/j.ajrs.20180601.11.

[29] P. K. Yadav, R. Srivastava, and N. Chaurasiya, "Optical Fiber Sensor: Review and Applications," International Journal of Creative Research Thoughts, vol. 6, no. 1, pp. 1358-1362, Mar. 2018.

[30] D. Ahuja and D. Parande "Optical Sensors and Their Applications," Journal of Scientific Research and Reviews, vol. 1, no. 5, pp. 60-68, 2012.

[31] P. H. Sureshkumar and R. Rajesh, "The Analysis of Different Types of IoT Sensors and security trend as Quantum chip for Smart City Management," IOSR Journal of Business and Management (IOSR-JBM), vol. 20, no. 1, pp. 55-60, Jan. 2018, doi: 10.9790/487X-2001045560. 
[32] N. Sabri, S. Aljunid, M. Salim, R. B. Ahmad, and R. Kamaruddin, "Toward Optical Sensors: Review and Applications,” J. Phys. Conf. Ser., vol. 423, p. 2064, Apr. 2013, doi: 10.1088/1742-6596/423/1/012064.

[33] O. Vermesan and P. Friess, Digitising the Industry - Internet of Things Connecting the Physical, Digital and Virtual Worlds, Publishers, 2016, doi: 10.13052/rp-9788793379824.

[34] G. Luka et al., "Microfluidics Integrated Biosensors: A Leading Technology towards Lab-on-a-Chip and Sensing Applications," Sensors (Basel)., vol. 15, no. 12, pp. 30011-30031, Dec. 2015, doi: 10.3390/s151229783.

[35] K. Weir and J. D. C. Jones, "Optical Fiber Sensors: Optical Detection BT - Optical Fiber Sensor Technology: Fundamentals," Eds. Boston, MA: Springer US, pp. 293-325, 2000.

[36] A. H. Zaidi, R. Roncella, and L. Zeni, "Advanced Dynamic Interrogation Techniques for Hybrid Distributed/Discrete Optical Fiber Sensors," 2013

[37] W. R. Habel, "Optical Fiber Methods in Nondestructive Evaluation BT - Handbook of Advanced Nondestructive Evaluation," N. Ida and N. Meyendorf, Eds. Cham: Springer International Publishing, pp. 595-642, 2019.

[38] G. Gauglitz, "Direct Optical Sensors: Principles and Selected Applications," Anal. Bioanal. Chem., vol. 381, pp. 141-155, Feb. 2005, doi: 10.1007/s00216-004-2895-4.

[39] Y. R. García, J. M. Corres, and J. Goicoechea, "Vibration Detection Using Optical Fiber Sensors," J. Sensors, vol. 2010, p. 936487, 2010, doi: 10.1155/2010/936487.

[40] B. Pal, "Optical Fiber Sensors: A Versatile Technology Platform for Sensing," J. Indian Inst. Sci., vol. 94, pp. 284-309, Nov. 2014.

[41] A. R. Bahrampour, M. Bathaee, S. Tofighi, A. Bahrampour, F. Farman, and M. Vali, "Polarization maintaining optical fiber multi-intruder sensor," Opt. Laser Technol., vol. 44, no. 7, pp. 2026-2031, 2012, doi: 10.1016/j.optlastec.2012.03.032.

[42] O. S. Wolfbeis and B. M. Weidgans, "Fiber Optic Chemical Sensors And Biosensors: A View Back," Optical Chemical Sensors. Springer Netherlands, Dordrecht, pp. 17-44, 2006, doi: 10.1007/1-4020-4611-1_2.

[43] R. Correia, S. James, S.-W. Lee, S. P. Morgan, and S. Korposh, "Biomedical application of optical fibre sensors," J. Opt., vol. 20, no. 7, p. 73003, 2018, doi: 10.1088/2040-8986/aac68d.

[44] R. Y. Shah and Y. K. Agrawal, "Introduction to fiber optics: Sensors for biomedical applications," Indian J. Pharm. Sci., vol. 73, no. 1, pp. 17-22, Jan. 2011, doi: 10.4103/0250-474X.89752.

[45] L. Chin, W. Whelan, and I. Vitkin, "Optical Fiber Sensors for Biomedical Applications," in Optical-Thermal Response of LaserIrradiated Tissue, pp. 661-712, 2011, doi: 10.1007/978-90-481-8831-4_17.

[46] D. Tosi, S. Poeggel, I. Iordachita, and E. Schena, "11 - Fiber Optic Sensors for Biomedical Applications," Opto-Mechanical Fiber Optic Sensors. Butterworth-Heinemann, pp. 301-333, 2018, doi: 10.1016/B978-0-12-803131-5.00011-8.

[47] C. P. Mayoral et al., "Fiber Optic Sensors for Vital Signs Monitoring. A Review of Its Practicality in the Health Field," Biosensors, vol. 11, no. 2, p. 58, 2021, doi: 10.3390/bios11020058.

[48] R. Sante, "Fibre Optic Sensors for Structural Health Monitoring of Aircraft Composite Structures: Recent Advances and Applications," Sensors (Basel)., vol. 15, pp. 18666-18713, Aug. 2015, doi: 10.3390/s150818666.

[49] C. S. Monteiro, M. Raposo, P. A. Ribeiro, S. O. Silva, and O. Frazão, "Acoustic Optical Fiber Sensor Based on Graphene Oxide Membrane," Sensors, vol. 21, no. 7, p. 2336, 2021, doi: 10.3390/s21072336.

[50] B. Lee, "Review of the present status of optical fiber sensors," Opt. Fiber Technol., vol. 9, no. 2, pp. 57-79, 2003, doi: $10.1016 / \mathrm{S} 1068-5200(02) 00527-8$

\section{BIOGRAPHY OF AUTHOR}

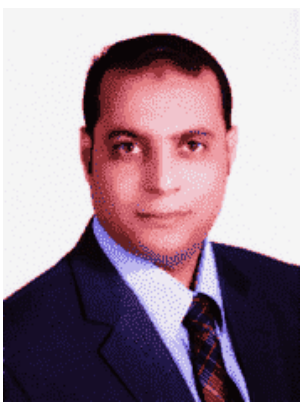

Dr. Mahmoud M. A. Eid (1) If EC P was born in Gharbiya State, Egypt, in 1977. Received a B.Sc., M.Sc., Ph.D. degrees in the Electronics Communication Engineering Department from the Faculty of Electronic Engineering, Menoufia University in 2002, 2007, and 2012 respectively. Currently, He joins now Taif University as an Assistant Professor in the Electrical Engineering Department, Faculty of Engineering at Taif University (KSA). His scientific master science thesis has focused on optical amplifiers in optical access communication systems. His interesting research mainly focuses on optical sources, amplifiers, detectors, sensors, and optoelectronic devices. He can be contacted at email: m.elfateh@tu.edu.sa. 